Louisiana State University

LSU Digital Commons

$9-1-2007$

\title{
Crossover from Landau Fermi liquid to non-Fermi liquid behavior: Indications from Hall measurements on CeColn5
}

\author{
C. Capan
}

Max Planck Institute for Chemical Physics of Solids

S. Singh

Max Planck Institute for Chemical Physics of Solids

S. Nair

Max Planck Institute for Chemical Physics of Solids

M. Nicklas

Max Planck Institute for Chemical Physics of Solids

H. Lee

University of California, Irvine

See next page for additional authors

Follow this and additional works at: https://digitalcommons.Isu.edu/physics_astronomy_pubs

\section{Recommended Citation}

Capan, C., Singh, S., Nair, S., Nicklas, M., Lee, H., DiTusa, J., Fisk, Z., Wirth, S., \& Steglich, F. (2007).

Crossover from Landau Fermi liquid to non-Fermi liquid behavior: Indications from Hall measurements on CeColn5. Physica C: Superconductivity and its Applications, 460-462 I (SPEC. ISS.), 678-679.

https://doi.org/10.1016/j.physc.2007.03.175

This Article is brought to you for free and open access by the Department of Physics \& Astronomy at LSU Digital Commons. It has been accepted for inclusion in Faculty Publications by an authorized administrator of LSU Digital Commons. For more information, please contact ir@lsu.edu. 
Authors

C. Capan, S. Singh, S. Nair, M. Nicklas, H. Lee, J. F. DiTusa, Z. Fisk, S. Wirth, and F. Steglich 


\section{UC Irvine}

\section{UC Irvine Previously Published Works}

\section{Title}

Crossover from Landau Fermi liquid to non-Fermi liquid behavior: Indications from Hall measurements on CeColn5

\section{Permalink}

https://escholarship.org/uc/item/69h8f4g8

\section{Journal}

Physica C: Superconductivity and its Applications, 460-462 I(SPEC. ISS.)

\section{ISSN}

0921-4534

\section{Authors}

Capan, C

Singh, $S$

Nair, S

et al.

\section{Publication Date}

2007-09-01

\section{DOI}

10.1016/j.physc.2007.03.175

\section{License}

https://creativecommons.org/licenses/by/4.0/4.0

Peer reviewed 


\title{
Crossover from Landau Fermi liquid to non-Fermi liquid behavior: Indications from Hall measurements on $\mathrm{CeCoIn}_{5}$
}

\author{
C. Capan ${ }^{\text {a,b }}$, S. Singh ${ }^{a, 1}$, S. Nair ${ }^{\text {a }}$, M. Nicklas ${ }^{\text {a }}$, H. Lee ${ }^{\text {c }}$, J.F. DiTusa ${ }^{b}$, \\ Z. Fisk ${ }^{\mathrm{c}}$, S. Wirth ${ }^{\mathrm{a}, *}$, F. Steglich ${ }^{\mathrm{a}}$ \\ ${ }^{a}$ Max Planck Institute for Chemical Physics of Solids, Nöthnitzer Straße 40, 01187 Dresden, Germany \\ ${ }^{\mathrm{b}}$ Department of Physics and Astronomy, Louisiana State University, Baton Rouge, LA 70803, USA \\ ${ }^{\mathrm{c}}$ Department of Physics, University of California, Irvine, CA 95616, USA
}

Available online 28 March 2007

\begin{abstract}

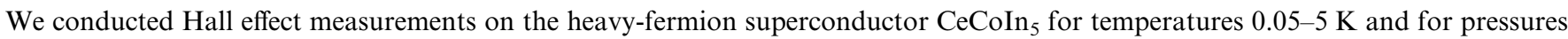
up to $1.2 \mathrm{GPa}$. A scaling of the magnetic field $H$ is introduced for the differential Hall coefficient, $R_{\mathrm{H}}^{\mathrm{d}}=\partial \rho_{x y}(T, H) / \partial H$ resulting in a single generic curve for $R_{\mathrm{H}}^{\mathrm{d}}(H)$ curves obtained at different $T$. We argue that the peak feature apparent in this generic curve corresponds to the crossover from non-Fermi liquid to Landau Fermi liquid behavior.
\end{abstract}

(c) 2007 Elsevier B.V. All rights reserved.

PACS: 74.70.Tx; 75.47.- $\mathrm{m}$

Keywords: Superconductivity; Heavy-fermion metal; Landau Fermi liquid

The compound $\mathrm{CeCoIn}$ is particularly suited to investigate the interplay of quantum criticality and unconventional superconductivity (SC) in which the pairing might be mediated by magnetic fluctuations. It exhibits the highest superconducting critical temperature, $T_{\mathrm{c}}$, among the Ce-based ambient pressure superconductors [1], a magnetic field tuned quantum critical point (QCP) may exist [2] close to the upper critical field of SC, $H_{\mathrm{c} 2}$, and $\mathrm{SC}$ may hide an antiferromagnetic (AFM) order.

Hall effect measurements are a well established tool to shed light on the electronic properties of materials close to a QCP. Accordingly, such measurements have early been conducted for $T \geqslant 1 \mathrm{~K}$ [3], even for applied pressures $p$ [4]. In our case, we want to concentrate on the low- $T$ region $0.05 \mathrm{~K} \leqslant T \leqslant 5 \mathrm{~K}$ and $p \leqslant 1.2 \mathrm{GPa}$. At these $T$ well

\footnotetext{
${ }^{*}$ Corresponding author. Tel.: +49 3514646 3229; fax: +49 3514646 3232.

E-mail address: wirth@cpfs.mpg.de (S. Wirth).

${ }^{1}$ Present address: Laboratoire de Physico-Chimie de l'Etat Solide, Université Paris-Sud, 91405 Orsay, France.
}

below the coherence temperature $T^{*} \approx 40 \mathrm{~K}$ no anomalous Hall contribution is found. However, interpretation of Hall effect in $\mathrm{CeCoIn}_{5}$ is complicated since SC inhibits a determination of the initial Hall coefficient and multiple bands at the Fermi level contribute to the Hall signal with a field dependent cyclotron mass [5].

For Hall measurements, isothermal field sweeps were conducted on single crystalline $\mathrm{CeCoIn}_{5}$ samples with $H \| c$. Measurements under pressure were carried out in a piston cylinder type pressure cell. The evolution of the Hall resistivity $\rho_{x y}$ (left) and its differential $R_{\mathrm{H}}^{\mathrm{d}}=\partial \rho_{x y} / \partial H$ (right) for increasing $p$ at $T=120 \mathrm{mK}$ is shown in Fig. 1. A changing slope of $\rho_{x y}(H)$, as obvious from the $T=120 \mathrm{mK}$ data, is observed for $0.1 \leqslant T \leqslant 0.3 \mathrm{~K}$ at $p=0$ and $0.3 \mathrm{GPa}$ resulting in a minimum of $\left|R_{\mathrm{H}}^{\mathrm{d}}\right|$ (arrow). This feature is suppressed with increasing $p$ and can no longer be resolved at $1.2 \mathrm{GPa}$.

For further analysis, the $H$-values of the ambient pressure isothermal $R_{\mathrm{H}}^{\mathrm{d}}(T, H)$ vs. $H$ curves were scaled by $H_{\text {min }}^{\mathrm{d}}$. Here, $H_{\min }^{\mathrm{d}}$ denotes the field value at which $\left|R_{\mathrm{H}}^{\mathrm{d}}\right|$ assumes its minimum for $70 \leqslant T \leqslant 200 \mathrm{mK}$. As seen in 


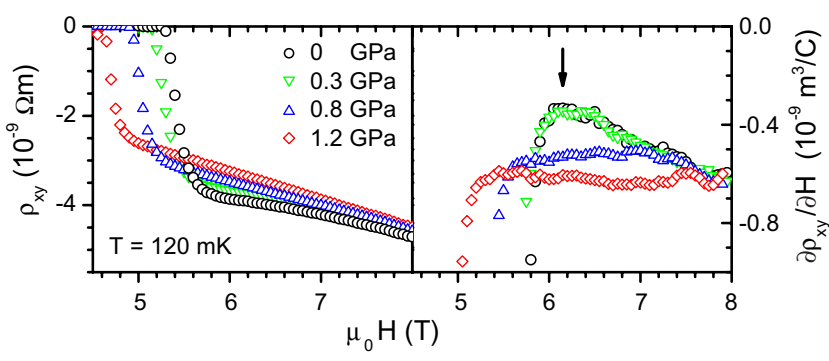

Fig. 1. Evolution of the isothermally measured Hall resistivity $\rho_{x y}$ (left) and differential Hall coefficient $\partial \rho_{x y} / \partial H$ (right) of $\mathrm{CeCoIn}_{5}$ with pressure at $120 \mathrm{mK}$. The arrow indicates the "peak feature" referred to in the text.

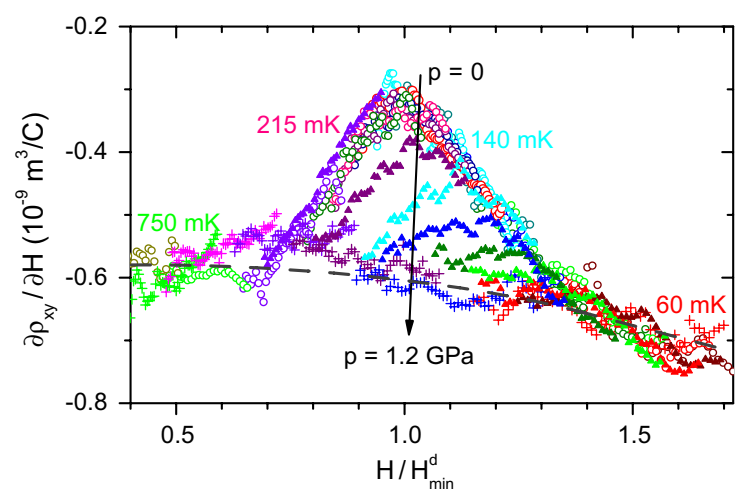

Fig. 2. Summarized differential Hall coefficient for $p=0(\bigcirc), 0.8 \mathrm{GPa}(\boldsymbol{\Delta})$ and $1.2 \mathrm{GPa}(+)$ illustrating the increasing suppression of the Hall "peak feature" (arrow in Fig. 1). Magnetic fields are scaled with respect to $H_{\min }^{\mathrm{d}}$ and different temperatures are presented by different colors.

Fig. 2(O), all scaled $R_{\mathrm{H}}^{\mathrm{d}}$ curves collapse onto a single, generic curve. For $T$ below/above the given range, the $H$-values were scaled such that this generic curve is further completed towards larger/smaller values $H / H_{\min }^{\mathrm{d}}$. Here, $\mu_{\text {eff }} \propto 1 / H_{\min }^{\mathrm{d}}$ can be viewed as effective mobility, averaged over all Fermi surfaces (FS) contributing.

The ambient pressure $R_{\mathrm{H}}^{\mathrm{d}}$ data between $0.7 \leqslant H / H_{\min }^{\mathrm{d}} \leqslant$ 1.3 mark a distinct "peak feature", whereas smaller and larger scaled fields appear to form an underlying "base line" of weak $H$-dependence (dashed line, Fig. 2). This "peak feature" is likely related with the AFM spin fluctuations (SF), based on the following observations:

(i) $p$ dependence: Applying pressure to $\mathrm{CeCoIn}_{5}$ drives the system towards a heavy Landau Fermi liquid (LFL) state [6] by gradually suppressing the AFM SF [7]. This is likely related to the increase of $T_{\mathrm{c}}$ with $p$ for small $p$, leading to maximum $T_{\mathrm{c}}$ at $p^{*} \sim 1.3 \mathrm{GPa}$. Our $R_{\mathrm{H}}^{\mathrm{d}}$ data at $p=1.2 \mathrm{GPa}(+$,
Fig. 2), i.e. slightly below $p^{*}$, approach the base line, with minor deviations at $H / H_{\min }^{\mathrm{d}} \sim 0.7$. For intermediate $p=0.8 \mathrm{GPa}$, the $R_{\mathrm{H}}^{\mathrm{d}}$ values appear to be reduced for lower fields only. Note that for $H$ scaling at $p>0$ the $H_{\min }^{\mathrm{d}}$ values obtained at $p=0$ were used. (ii) $R_{\mathrm{H}}^{\mathrm{d}}$ values: At low $H\left(<0.7 H_{\min }^{\mathrm{d}}\right)$ we obtain $-R_{\mathrm{H}}^{\mathrm{d}} \approx 6 \times 10^{-10} \mathrm{~m}^{3} / \mathrm{C}$ with a slight $H$ dependence $\left(7 \times 10^{-10} \mathrm{~m}^{3} / \mathrm{C}\right.$ at $\left.1.5 H_{\min }^{\mathrm{d}}\right)$. This value agrees remarkably well with the one reported [4] for the non-magnetic analogue $\operatorname{LaCoIn}_{5}\left(-5.5 \times 10^{-10} \mathrm{~m}^{3} /\right.$ C). Generally, pressure drives Ce from a $4 \mathrm{f}^{1}$ towards a non-magnetic $4 \mathrm{f}^{0}$ configuration. Moreover, $R_{\mathrm{H}}^{\mathrm{d}}$ of the Ce- and the La-based compound agree well at $\mu_{0} H=7 \mathrm{~T}$, i.e. in the LFL regime.

(iii) The $T$ dependence of $H_{\min }^{\mathrm{d}}$ as obtained from the scaling (Fig. 2) tracks the crossover [2] from non-Fermi liquid to LFL behavior (not shown).

The "peak feature" might be related to AFM SF or to the opening of an AFM gap at the FS if a spin density wave is formed. The latter may also cause a discontinuity in $R_{\mathrm{H}}^{\mathrm{d}}$ [8]. However, pressure suppresses the "peak feature" while changing the FS only little [5]. Note that Hall measurements (unlike thermodynamic ones) are sensitive to even weak fluctuations. Hence, the anisotropic AFM SF might be considered as a precursor of a gap opening.

\section{Acknowledgments}

S.W. and M.N. are partially supported by the EC, CoMePhS 517039 and the DFG through SFB 463, respectively. S.N. and Z.F. are supported by the Humboldt Foundation. H.L. and J.F.D. acknowledge support by the NSF through Grants DMR 0533560 and DMR 04 06140, respectively.

\section{References}

[1] C. Petrovic et al., J. Phys.: Condens. Matter 13 (2001) L337.

[2] J. Paglione et al., Phys. Rev. Lett. 91 (2003) 246405;

A. Bianchi et al., Phys. Rev. Lett. 91 (2003) 257001.

[3] Y. Nakajima et al., J. Phys. Soc. Jpn. 73 (2004) 5; M.F. Hundley et al., Phys. Rev. B 70 (2004) 035113.

[4] Y. Nakajima et al., J. Phys. Soc. Jpn. 75 (2006) 023705.

[5] H. Shishido et al., J. Phys. Soc. Jpn. 71 (2002) 162;

T. Maehira et al., J. Phys. Soc. Jpn. 72 (2003) 854;

D. Hall et al., Phys. Rev. B 64 (2001) 212508.

[6] M. Nicklas et al., J. Phys.: Condens. Matter 13 (2001) L905; V.A. Sidorov et al., Phys. Rev. Lett. 89 (2002) 157004.

[7] M. Yashima et al., J. Phys. Soc. Jpn. 73 (2004) 2073.

[8] J. Fenton, A.J. Schofield, Phys. Rev. Lett. 95 (2005) 247201. 